\title{
Manejo y complicaciones en el tratamiento de la Atresia de Esófago
}

Pasaron casi 300 años desde la descripción de la atresia esofágica (AE) hasta corregir con éxito esta afección. Fue en 1939 cuando Leven con técnica en tres etapas, obtiene por primera vez sobrevida, luego C. Haight 10 consigue en 1941, corrigiendo el defecto en un solo tiempo $(1,2$.$) .$

Lo señalado indica gravedad importante, dado por la presencia de la fístula traqueoesofágica (FTE), por la asociación con otras malformaciones y prematurez.

En nuestro hospital, la primera reparación con éxito se efectú en 1962, y lo que en un comienzo estaba dirigido a obtener sobrevida, con el tiempo, al integrarse un equipo donde participan cirujanos, pediatras, anestesistas, enfermeras y auxiliares, abocados al problema de patología quirúrgica del recién nacido (RN), este éxito dejó de ser una sorpresa grata, transformándose en un hecho habitual, pues se logro solucionar las complicaciones que llevaban a la muerte, gracias al esfuerzo de cada uno y a la experiencia lograda.

\section{MATERIAL Y METODO}

Este materiai corresponde a la segunda revisión de $\mathrm{RN}$ con atresia esofágica operados en el Hospital Calvo Mackenna. El primer análisis se presentó en el Congreso de

- Servicio de Cirugía, Hospital Calvo Mackenna,

**Servicio de Prcmaturos, Hospital Calvo Mackenna.
Dres.: Ramón González LI.", J. Eduardo Caamaño M.*, Clara Román**, Inés Ebensperger."*

Cirugía Pediátrica de Viña del Mar, en 1970, que comprendió 38 casos tratados entre 1962-1970, con una sobrevida de 50\%.

Er nuestra casuística consideramos todos aquellos RN en que le cupo participación al cirujano, incluyéndose algunos casos en que sólo fue posible efectuar gastrostomía.

Todos nuestros enfermos ingresaron a la Unidad Médico-Quirúrgica de RN Patológico, del Centro de Prematuros del Hospital Calvo Mackenna. Un número importante provino de provincias, los que desgraciadamente siguen llegando después de muchas horas de vida.

Desde enero de 1971 a septiembre de 1975, ingresaron 47 RN con atresia de esófago (Tabla N: 1).

Tabla No 1

\section{ATRESIA ESOFAGICA. HOSPITAL CALVO} MACKENNA

Distribución por años

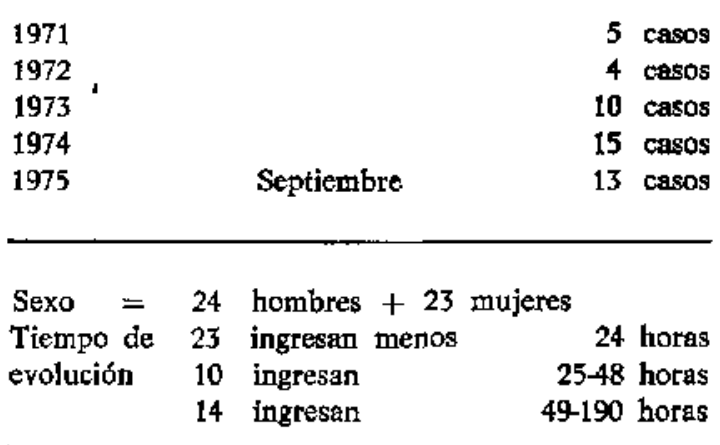




\section{DIAGNOSTICO}

El diagnóstico de la $A E$, es clínico. En un $\mathrm{RN}$, con salivación bucal importante, junto a signología respiratoria que puede confundirse con el síndrome de aspiración, la simple introducción por la boca de una sonda Nelaton 12-14 Fr, en buen estado, ayudará al diagnóstico. Si la sonda no pasa, hay atresia, y si el abdomen está distendido significa que hay fístula traqueoesofágica (FTE). Cuando el abdomen está excavado hay posibilidades gute no exista fístula.

Una radicgrafía tóraco-abdominal en frontal y lateral con la cabeza en extensión, es suficiente, ya que nos informa sobre la atresia, la presencia o ausencia de FTE, al existir o faltar aire en el addomen y el estado de los pulmones. El uso de medio de contraste, en el cabo superior, no es fundamental y puede ser dañino, si no se hace con precaución.

Creemos que en las maternidades y servicios de Neonatología no se debe pretender agotar el estudio, s6lo efectuar el pasaje de una sonda por boca y ante la sospecha, detivar a un centro especializado, sin perdet tiempo en confirmar la exactitud del diagnóstico, pues el enfriamiento, la acumulacion de secreciones, etc., perjudica el estado del $R N$ y retarda la intervención oportuna.

La distribución de nuestros casos, de acuerdo a la clasificación de Roberts, (3) se puede ver en la Tabla N: 2 y en la Figura No 1.

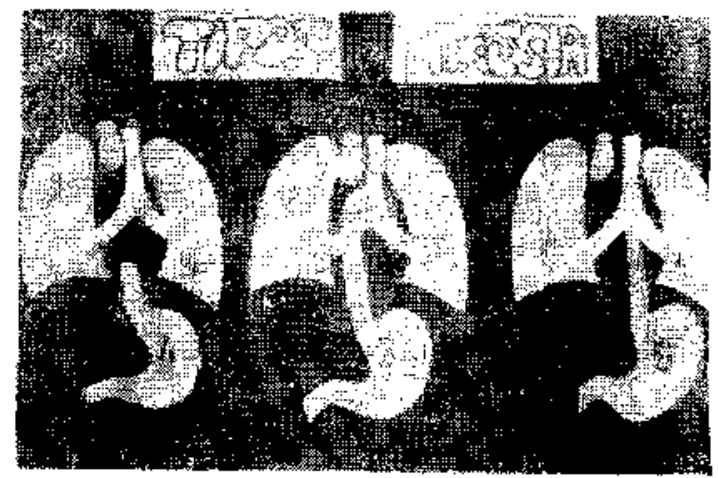

Figura N: 1
Tabla N: 2

ATRESIA ESOFAGICA (TIPOS)

\begin{tabular}{llrr}
\hline Tipo & & No casos & $\%$ \\
\hline Sin fistula & I & 4 & 8,5 \\
Con fistula & IIIA (Carina) & 9 & 19,1 \\
Con fístula & IIIB & 34 & 72,3 \\
\hline
\end{tabular}

Patología asociada (Tabla No 3). Prácticamente todos nuestros enfermos presentan al ingreso patologia pulmonar de variable magnitud (bronconeumonia, atelectasia, aspiración).

En $27,2 \%$ se encuentra antecedentes de hidroatanios.

Diecisiete casos $(36,1 \%)$ presentan anomalías congénitas asociadas, 8 de las cutales eran graves malformaciones, que inspidieron la operación correctora en 5 de ellos, siendo las cardiopatías congénitas y la trisomía 21 las más frecuentes.

Tabla $\mathrm{N}^{0} 3$

\section{ATRESIA DEL ESOFAGO. PATOLOGIA ASOCIADA}

7 cardiopatias (Fallot, CIV, CIV + CIA, etc.)

4 trisomías 21 ( 2 con cardiopatias)

3 fisuras palatinas (1 Jabio leporino)

2 imperforaciones anales

3 subelayias derechas aberrantes

1 agenesia pulmonar y dextrocardia

1 malformación lóbulo pulmonar derecho

I meningocele 1 hemivértebra

1 agenesia globo ocular 1 microftalmía

1 páncreas anular

1 tiñón en herradura

1 polislactilia

\section{MANEIO}

El primer concepto de importancia que planteamos, es evitar a toda costa la lesión de! parénquima pulmonar. El principal causante de este daño es la aspiración de jugo gástrico, de $\mathrm{pH}$ bajo, a través de la FTE a las vías aéreas, provocando lesiones cáusticas del árbol bronquial y alvéolos, donde actuarán posteriormente los gérmenes patógenos. 
Para evitarlo practicamos gastrostomía (Stamm) apenas ingresado el enfermo, lo que permite mejorar la mecánica respiratoria del $\mathrm{RN}$, evitar el mayor reflujo y cortegir alteraciones metabólicas, postergándose la urgencia y permitiendo intervenir con equipo anestêsico-quirúrgico adecuado y con el enfermo en las mejores condiciones posibles.

En la intervención definitiva, se cierra y secciona la fistula y se practica anastomosis término-terminal, en un solo tiempo, mediante toracotomía posterolateral derecha extrapleural, a nivel de la cuarta costilla. Usamos puntos separados de seda $5 / 0$, en el cierre de la fístula y en la anastomosis, la que hacemos en dos planos, uno de mucosa anudados en e! lumen, seguido de telescopaje del plano muscular. Drenaje sellado bajo trampa de agua.

El tratamiento postoperatorio, es el del RN quirúrgico, teniéndose especial cuidado en los siguientes puntos:

- Isolette con humedad máxima (Vapojette), para fluidificar las secreciones.

- Cambio frecuente de posición y percusión torácica.

- Estímulo del llanto y tos.

- Sonda de gastrostomía abierta.

- Aspiración bucofaríngeas de secreciones. cuidando no lesionar la anastomosis.

- Aspiración tráqueobronquial en caso necesario.

* Antibióticos de amplio espectro.

- Balance hidroelectrolítico y plasma.

Se inicia la alimentación por gastrostomía a las 48-72 horas de cerrada la fístula, en forma progresiva. Al $7^{\circ}$ día, tránsito esofágico y si hay buen pasaje, se inicia la alimentación oral. El drenaje se retira a las 72 horas. Antes del alta se practican calibraciones con bujías Philips, hasta llegar a $2426 \mathrm{Fr}$.

\section{COMPLICACIONES}

La alta mortalidad que presentó en los primeros años el tratamiento de la $\mathrm{AE}$, se debió en gran medida a las complicaciones postoperatorias, que siguen siendo frecuentes y algunas muy graves $(2,7)$. Nuestra experiencia tampoco es ajena a la alta incidencia de complicaciones, las que llegan a $54 \%$, siendo algunas de poca magnitud, pero que obligan al cirt1jano a un control estricto $y$ a veces a intervenir.

En 35 niños efectutamos la anastomosis; 16 hicieron una evolución excelente $(45.7 \%)$, el resto presentó problemas, llamando la atención el grupo cuya fístula nace a nivel de la carina.

Por su forma de aparición, clasificamos estas complicaciones en precoces y tardías. Están detalladas en la Tabla No 4.

Tabla N: 4

\section{COMPLICACIONES PRECOCES}

\begin{tabular}{lcc}
\hline Fistula esofágica & 7 & casos \\
Dificultad vaciamiento géstrico & 12 & " \\
Obstrucción intestinal (brida) & 1 caso \\
Perforación yeyunal & 1 & " \\
Vólvulo parcial de estómago & 1 & " \\
infecciones leves herida operatoria. &
\end{tabular}

La fístula o dehiscencia de la anastomosis, es la complicación precoz más importante por la frecuencia y la alta mortalidad que provoca (Tabla No 5).

Tabla N: 5

\section{ATRESIA DEL ESOFAGO. COMPLICACIONES} POSTOPERATORIAS

\begin{tabular}{lcc}
\hline Evolución de fístula esofágica & 7 & casos \\
Muertos & 3 & $n$ \\
Cierre espontáneo & 2 & " \\
Reoperado vivo & 1 caso \\
Peoperado esofagostomía & 1 & " \\
\hline
\end{tabular}

\section{DIFICULTAD EN EL VACIAMIENTO GASTRICO}

Ha llamado la atención que $12 \mathrm{RN}$ han presentado dificultad en el vaciamiento gástrico, lo que se advirtió en el depósito de la gastrostomía. El estudio radiológico mostró retario del vaciamiento, con píloro alargado y se consideró como estenosis pilórica atípica. Esto 
obligó a practicar piloromiotomías en 4 casos y una piloroplastía, sin encontrarse hipertrofia. Este problema se ha visto incluso en algunos RN que sólo tenían gastrostomía. No hemos encontrado descrito este hecho probablemente, porque otros autores no usan gastrostomía y no pueden apreciar tan claramente el tiempo de vaciamiento gástrico.

\section{PROBLEMAS DERIVADOS DE LA GASTROSTOMIA}

Esta operación, junto con beneficiar, ha traído 5 complicaciones: 1 obstrucción por brida, 1 oclusión con perforación yeyunal, y 1 vólvulo parcial de estómago. Estas dos últimas debido a la falta de fijación del estómago al peritoneo parietal, error que se vio en gastrostomías practicadas en otros servicios.

\section{ROTURA MASIVA DE ALVEOLOS}

Creemos necesario mencionar esta complicación evitable, producida accidentalmente, por hiperpresión en la ventilación durante la anestesia, que se evidenció por la aspiración bronquial hemorrágica, causando gran dificultad respiratoria, que obligó al uso prolongado de respirador automático y que contribuyó a la muserte.

\section{Tabla N: 6}

\section{ATRESIA DEL ESOFAGO. COMPLICACIONES} POSTOPERATORIAS TARDIAS
Estenosis postoperatorias

Bronquitis a repetición

Obstrueción par ingestión

de cuerpo extraño

Diverticulo esofágico

Desnutrición
7 casos

6 "

$3 "$

1 caso
Complicaciones tardías (Tabla $\mathrm{N}^{0}$ 6).

La más importante fue la estenosis postoperatoria qute se presentó en 7 niños (Tabla № 7).
Tabla No 7

\section{ATRESIA DEL ESOFAGO. ESTENOSIS POSTOPERATORIAS}

Por reflujo (retardo vaciamiento gástrico) 5 casos

Por falla de manejo de Enfermeria 2 "

Reoperados: 5 casos Dilataciones: 2 "

Es importante comentar que en el plazo de 1 mes se produjeron 3 estenosis completas, lo que coincidió con la llegada de nuevo personal auxiliar, sin experiencia en el manejo postoperatorio de estos pacientes y pensamos que la aspiración descuidada de secreciones bucales, con introducción de la sonda hasta la anastomosis, produjo retracción de la mucosa y cierre. Nos parece demasiada coincidencia que la mitad de las estenosis se hayan producido en un solo mes.

\section{BRONOUITIS A REPETICION}

Al igual que otros autores, (1) hemos observado en los primeros meses cuadros bronquiales a repetición; se han tabulado 6 , pero estimamos pueden ser más, por haber niños ctyo control se hace en provincias.

Tres pacientes de 8, 9 meses y 2 años, presentaron obstrucción del esófago por ingestión de cuerpo extraño o alimento, que se detuvieron en la zona que correspondería a la sutura, que se extrajeron por endoscopía.

\section{DIVERTICULO ESOFAGICO}

Se manifestó por problemas respiratorios a repetición (bronquitis y bronconeumonias), demostrándose en el tránsito, un divertículo de gran tamaño y que fue operado.

\section{DESNUTRICION}

En relación a malformaciones asociadas y bronquitis a repetición, se ha visto compromiso del desarrollo pondestatural que no hemos podido tabular. 


\section{RESULTADOS}

Siempre ha sido difícil analizar el tratamiento de esta afección en la que juegan factores como: malformaciones rongénitas asociadas, neumopatía, prematurez, que inciden en los resultados, por esto se han propuesto varias fórmulas de análisis. La Clasificación de Riesgo Operatorio, preconizada por Waterston en 1962, (5) por considerar estos factores, nos ha parecido la más racional y fácil (Tabla No: 8).

Taibla Ni 8

\section{CLASIFICACION RIESGO OPERATORIO}

Waterston, 1962

Grupo A-1 Peso nac. sobre 2.500 grs. $y$ bien

Grupo B-I Peso entre 1.800 y 2.500 grs. y bien

Grupo B-2 Buen peso neumopat. moderada $y$ anomal. congén, leve

Grupo C-1 Peso nac. bajo 1.800 grs.

Grupo C-2 Buen peso, neumopat. severa, grave.

En el análisis de resultados, no se incluyen $6 \mathrm{RN}$, en los que por las pésimas condiciones a su ingreso (cardiopatías, neumonias graves, o anomalias congénitas), sólo se pudo practicar gastrostomia, con la intención que mejoraran y pudieran llegar a la operación definitiva, lo que no ocurrió. Pensamos que en estos niños apenas se hizo una intervención preparatoria para la corrección de la atresia, por lo que se analizan solamente 41 casos.

La corrección de la $\mathrm{AE}$, se pretende siempre hacer en un tiempo, pero cuando hay por lo menos dos de las siguientes condiciones: (6) prematurez, bajo peso, anomalías congénitas importantes, neumonia severa, bitirrubina elevada, problemas anatómicos (cabos distantes $y / o$ friables), problemas de pabellón (equipo médico-quirúrgico) y en la atresia sin fistula, deberá hacerse tratamiento en etapas.

El tratamiento de nuestros 41 casos se efectuó en etapas en $11, \mathrm{y}$ en 30 en un tiempo.
Table N: 9

\section{ATRESIA DEL ESOFAGO. TRATAMIENTO EN ETAPAS}

\begin{tabular}{|c|c|c|}
\hline Atresia esofágica sin fístula & 4 & chsos \\
\hline Gastrostomía (malformaciones) & 2 & mutertos \\
\hline $\begin{array}{l}\text { Gastrostomía, esofagostomía } \\
\text { (5 y } 6 \text { meses) }\end{array}$ & 2 & muertos \\
\hline Atresio esofógica con fistula & 7 & casos \\
\hline Gastrostomía y ligadura fístula & 2 & muertos \\
\hline Gastrost., ligadura, esofagostomía & 1 & vivo \\
\hline Gastrost. y ligadura. Anastomosis & 4 & vivos \\
\hline
\end{tabular}

De acuerdo con la Clasificación de Waterston, nuestros resultados yan en la Tabla No 10.

Tabla No 10

ATRESIA DEL ESOFAGO. CLASIFICACION DE RIESGO OPERATORIO

\begin{tabular}{lcccc}
\hline & N: & Muertos & Vivos & $\%$ \\
\hline & & & & \\
Grupo A & 13 & 0 & 13 & 100 \\
Grupo B & 15 & 7 & 8 & $53,3^{*}$ \\
Grupo C & 13 & - & 6 & $46,5^{* * 4}$ \\
\cline { 2 - 5 } & 41 & 14 & 27 & \\
\hline
\end{tabular}

*La sobrevida del Grupo B aumenta a $66,6 \%$ si se excluyen los 6 casos en que no se practicó la anastomosis.

**Grupo C aumenta a $60 \%$.

Sobrevitia general $77,1 \%$

(35 corregidos: 27 vivos y 8 muertos)

\section{DIAS DE HOSPITALIZACION}

Cuando la evolución fue satisfactoria la hospitalización alcanzó un promedio de 41,2 días (26 a 60 días). La operación en etapas y las complicaciones la elevaron a 87 días, con extremas que van de $\operatorname{los} 63$ a 120 días. 


\section{ANALISIS DE LA MORTALIDAD} (Tabla N? 11)

Es importante mencionar que las 4 atresias sin fístula murieron 2 de ellas por las malformaciones, siendo posible sólo la gastrostomía, y los otros 2 que fallecen a los 5 y 6 meses por diarrea.

Tabla N: 11

ATRESIA DEL ESOEAGO. MORTALIDAD EN CORRECCION EN UN TIEMPO

Fistula postoperatoria 3 casos

1 con malformaciones asociadas

1 más rotura masiva de alvéolos

Reintervención estenosis esofágica

1 caso

Trisomía 21 + CIV amplio, muere a 49 días de primera operación

Decurarizución incompleto

2 casos

Patos respiratorios repetidos, mueren a 15 y 16 horas.

\section{Hemorragia intracraneana? Cardiopatía?}

2 casos

Convulsiones y cianosis (no tienen anat. patológica).

\section{COMENTARIOS}

Nos parece fundamental considerar esta afección como emergencia médico-quirúrgica, por lo tanto se deberán adoptar todas las medidas, para que desde la sospecha diagnóstica el médico se preocupe de disponer de su traslado en las mejores condiciones posibles. Si fuera de provincias alejadas de centros quirúrgicos de $\mathrm{RN}$, practicar gastrostomía y trasladar con todos los cuidados recomendados del RN Patológico.

Al igual que otras revisiones, la fístula y la estenosis postoperatorias son las complicaciones más importantes y graves que ensombrecen el pronóstico.

Creemos que nuestros resultados son alen* tadores, pero debemos superar problemas co- mo son: la atresia sin fistula y la $\mathrm{AE}$ con fístula a nivel de la carina, la primera alcanza a $8,5 \%$, debe repararse obligatoriamente en etapas y no hemos podido llegar a una edad o peso adecuado, para la operación de transposición de colon. La fístula que nace en la carina, con su cabo distal más corto y delgado, hace más difícil la anastomosis y facilita el reflujo y aspiración de contenido gástrico al árbol bronquial. En nuestra serie alcanza a 19,1\% y significó que 3 casos no alcanzaran a corregirse y 3 se complican (fístula, divertículo, estenosis).

Quisiéramos realzar un hecho, que debiera imitarse en todos los servicios: si algún éxito hemos tenido, ha sido posible en gran medida, a que en nuestro hospital existe un equipo médico-quirúrgico $\mathrm{y}$ se ha creado la Unidad de RN Patológico con enfermetas y auxiliares que han alcanzado una alta técnica y experiencia, que hace innecesario el uso de monitoraje en los cuidados postoperatorios de los enfermos.

\section{RESUMEN}

1. Se analizan 47 casos de atresia esofágica, atendidos en el Hospital Calvo Mackenna, entre 1971 y septiembre de 1975.

2. $36,1 \%$ de nuestros enfermos presenta malformaciones congénitas agregadas, predominando las cardiopatías y la trisomía 21.

3. Se comunica la metódica seguida en su tratamiento tanto en el pre, trans y postoperatorio.

4. La corrección en un tiempopo, se practicó en 30 casos, en 11 se hace en etapas, 6 de los cuales mueren antes de practicar la anastomosis.

5. Más del $50 \%$ de los casos presenta algún tipo de complicación, siendo la fístula y la estenosis postoperatoria, las más importantes, tanto por su frecuencia como por su gravedad. Hemos observado un retardo en el vaciamiento gástrico, sin comprender aún el mecanismo de su producción.

6. La atrosia sin fistula, constituye un problema en nuestro medio. La atresia con 
fístula que nace de la carina, es frecuente $(19,1 \%)$ y de tnal pronóstico.

7. Se analizan los resultados, siguiendo la Clasificación de Riesgo Operatorio de Waterston, destacando que frente a un nifio en buenas condiciones y sin malformaciones, la sobrevida alcanza a $100 \%$.

\section{SUMMARY}

1. 47 cases of Oesophagcal atresia treated at L. Calvo Mackenna children's Hospital (Stgo.-Chile) from 1971-1975 (Septiembre), are reported.

2. Aditional congenital Abnormalities were seen in $36,1 \%$ of our patients Cardiopaties and Down sindrome being the most frequent finding.

3. Pre, Trans and Postoperative procedures are described.

4. Primary anastomosis was perfomed in 30 cases, 11 patients required staged procedisres, 6 of these died before anastomosis could be undertaken.
5. Over half of patients developed complications mostly anastomosis leak and strictures. Minot complications consisted in delayed gastric emptying and recurrent Bronchitis.

6. Atresia without fistula is a problem for us, Atresia IIIA (fistula in carina) have a bad prognosis.

7. Accordingly with Waterston Clasification, group A showed an excellent prognosis and a survival rate of $100 \%$.

\section{REFERENCIAS}

1. El Shafie, M, and P. P. Rickham: Kinder chjrurgie $93 / 4,1971$.

2. Livaditis, A.: Scandinavian Journal of thoracic and cardiovascula surgery Suplement 2, 1969.

3. Freeman, N. V.: Neonatal Surgery. Rickham. P. P.. J. H. Johnston London Butterworths, 1969.

4. Eklöl. O. Livaditis, A. and Okmian, L.: Annales de Radiologuie 13 (3-4), 298-302, 1970.

5. Waterston, D. I., R. E. Bonham Carter and $E$. Aberden: Lancet 1: 819-822, 1962.

6. Feins, Neil R.: Ped. Clin. of N. A. 21 (2): 361 378, May 1974.

7. Koop, Everett: Progress in pediatric surgery 2: 41.56, 1971. 